\title{
Testing Models with Brown Dwarf Binaries
}

\author{
Trent J. Dupuy and Michael C. Liu \\ Institute for Astronomy, University of Hawai'i \\ 2680 Woodlawn Drive, Honolulu, HI, USA
}

\begin{abstract}
We have been using Keck laser guide star adaptive optics to monitor the orbits of ultracool binaries, providing dynamical masses at lower luminosities and temperatures than previously available and enabling strong tests of theoretical models. (1) We find that model color-magnitude diagrams cannot reliably be used to infer masses as they do not accurately reproduce the colors of ultracool dwarfs of known mass. (2) Effective temperatures inferred from evolutionary model radii can be inconsistent with temperatures derived from fitting observed spectra with atmospheric models by at most 100-300 K. (3) For the single pair of field brown dwarfs with a precise mass $(3 \%)$ and age determination $(\approx 25 \%)$, the measured luminosities are $\sim 2-3 \times$ higher than predicted by model cooling rates (masses inferred from $L_{\mathrm{bol}}$ and age are 20$30 \%$ larger than measured). Finally, as the sample of binaries with measured orbits grows, novel tests of brown dwarf formation theories are made possible (e.g., testing theoretical eccentricity distributions).
\end{abstract}
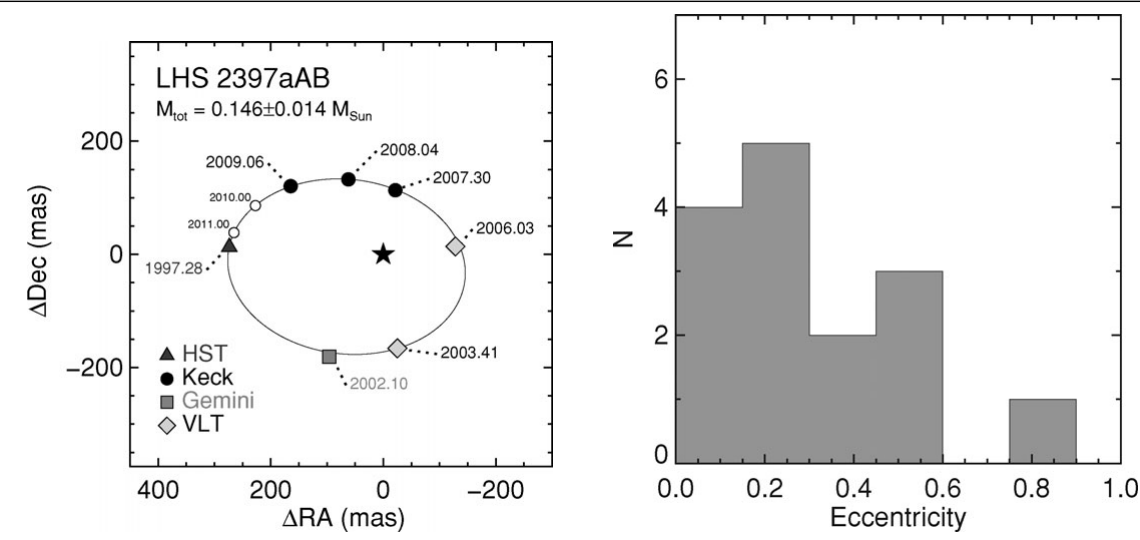

Figure 1. Left: Orbit of the M8+L7 binary LHS 2397aAB. Right: Eccentricity distribution for all nine published ultracool $(>\mathrm{M} 6)$ binary orbits along with six unpublished orbits known to the author.

Left:Dupuy et al. (2009b) Dupuy, Liu, \& Ireland

Right: Bouy et al. (2004) Bouy, Brandner, Martín, Delfosse, Allard, Baraffe, Forveille, \& Demarco, Simon et al. (2006) Simon, Bender, \& Prato, Seifahrt et al. (2008) Seifahrt, Röll, Neuhäuser, Reiners, Kerber, Käufl, Siebenmorgen, \& Smette, Liu et al. (2008) Liu, Dupuy, \& Ireland, Blake et al. (2008) Blake, Charbonneau, White, Torres, Marley, \& Saumon, Cardoso et al. (2009) Cardoso, McCaughrean, King, Close, Scholz, Lenzen, Brandner, Lodieu, \& Zinnecker, Dupuy et al. (2009a) Dupuy, Liu, \& Ireland, Dupuy et al. (2009b) Dupuy, Liu, \& Ireland, Dupuy et al. (2009c) Dupuy, Liu, \& Bowler

\section{References}

Blake, C. H., et al. 2008, Ap. Lett., 678, L125

Bouy, H., et al. 2004, A\& $A, 424,213$

Cardoso, C. V., et al. 2009, in AIP Conf. Ser. 1094, ed. E. Stempels, 509-512 
Dupuy, T. J., Liu, M. C., \& Ireland, M. J. 2009b, ApJ, 692, 729 -. 2009c, ApJ, 699, 168

Dupuy, T. J., Liu, M. C., \& Bowler, B. P. 2009a, ApJ, in press (astro-ph/0909.4784)

Liu, M. C., Dupuy, T. J., \& Ireland, M. J. 2008, ApJ, 689, 436

Seifahrt, A. et al. 2008, A\&SA, 484, 429

Simon, M., Bender, C., \& Prato, L. 2006, ApJ, 644, 1183 